\title{
Einfluss des Energie- und Proteinniveaus sowie der Proteinqualität auf die Milchleistung der Bunten Deutschen Edelziege
}

\begin{abstract}
Summary
Title of the paper: Influence of energy level and dietary protein quality and quantity on the lactation performance of German Fawn Goats

In this study two feeding trials were conducted using a total of 45 goats (German Fawn) to estimate milk yield for a lactation period of 150 days. Goats were fed corresponding German (high, H) and International (low, N) feeding standards for milk production. At each feeding level the diet had either protected (P, formaldehyde) or unprotected (U) Soya meal, resulting in different UDP amounts. The diets used in the trials had different protein contents: V1 low (11.9- 12.3\%) and V2 high (15.9-16.6\%) respectively. The diet were composed of hay and pellets with energy contents ranging from 11.3 to $11.6 \mathrm{MJ}$ ME/ kg DM. The milk yield was $40 \%$ lower in groups of V1 as compared to groups of V2 which is mainly attributed to the decreased energy and protein intake, partly caused due to low feed intake and the lack of RDP for optimal rumen microbial activity in V1. There also existed a strong correlation between feed intake and protein content of the diet. Feeding of protein protection resulted in an increased protein amount at duodenum and the goats gave a higher milk yield, provided a sufficient RDP level was secured

A high protein content in diet (16\%) at middle and late lactation induced a protein surplus which is combined with inefficient energy utilisation. During the lactation period the diets have to be adapted not only for energy but also for protein needs
\end{abstract}

Key Words: lactating goats, feeding, requirements, protein protection, milk yield,

\section{Zusammenfassung}

In zwei Versuchen standen insgesamt 45 laktierende Ziegen der Rasse Bunte Deutsche Edelziege über die ersten 150 Laktationstage für die Prüfung der Milchleistung zur Verfügung. Die Tiere erhielten entsprechend der deutschen und internationalen Normen für die Milchleistung ein hohes $(\mathrm{H})$ bzw. niedriges $(\mathrm{N})$ Energieangebot. Jede Gruppe wurde in zwei Untergruppen geteilt, die durch Gabe von ungeschütztem (U) bzw. geschütztem (P) Sojaextraktionsschrot unterschiedliche Anteile an im Pansen unabbaubarem Protein erhielten. Die beiden Versuche unterschieden sich durch einen niedrigen (11,9-12,3\%) bzw. hohen (15,9 bis 16,6\%) Rohproteingehalt der Ration. Die Diät bestand aus Wiesenheu und einem pelletierten Konzentratfutter mit veränderter Komponentenzusammensetzung, jedoch ausgeglichenem Energiegehalt (11,2 bis 11,6 MJ ME/ kg TS).

Die Milchleistung im ersten Versuch war um 40\% geringer als im zweiten Versuch. Ursache dafür waren die geringere Energie- und Proteinaufnahme, die z.T. durch reduzierte Futteraufnahme hervorgerufen wurde. Es bestand ein enger Zusammenhang zwischen Proteingehalt und Futteraufnahme. Weiterhin führte der niedrige Proteingehalt im V1 zu einem Mangel an RDP, sogar bei Einsatz von ungeschütztem Protein (Gruppe U), der nicht durch den Stoffwechselweg der N-Rezyklierung ausgeglichen werden konnte. Stickstoffmangel im Pansen, bzw. zu geringe Verfügbarkeit des Stickstoffs während der Phase der höchsten Energiefreisetzung führte zu einer Überversorgung mit Energie.

Durch den Proteinschutz wurde das Proteinangebot am Duodenum erhöht. Damit verbunden war eine Vergrößerung des Quotienten MP:ME, was eng mit einer höheren Milchleistung korrelierte. Bei höherem Proteingehalt in der Ration (16\%) ist besonders in der Mittel- und Spätlaktation ein Proteinüberschuß gegeben, was zu einer ineffizienten Energienutzung führt. Während der Laktation ist auf den veränderten Bedarf an Energie und Protein zu reagieren.

Schlüsselwörter: Milchziegen, Fütterung, Bedarfsnormen, Proteinschutz, Milchleistung 


\section{Einleitung}

Die Bedeutung der Milchziegenhaltung steigt weltweit und hat in den letzten Jahren auch in Europa deutlich zugenommen.

In den entwickelten Ländern sank in den letzten 15 Jahren die Produktion von Kuhmilch kontinuierlich (-4,9\%), dagegen stieg die Milchproduktion aus der Schaf$(+11 \%)$ und Ziegenhaltung (+21\%) beträchtlich an (MORAND-FEHR und BOYAZOGLOU, 1999).

Trotz wachsender Bedeutung der Ziegenmilchproduktion scheint das Leistungspotenzial der Ziegen nicht vollständig genutzt zu werden. Kenntnisse über Fütterung und Haltung der Ziegen sind im Vergleich zu dem Wissen bei Rindern lückenhaft (MORAND-FEHR und BOYAZOGLOU, 1999).

Während in Ländern mit intensiver Ziegenhaltung Fütterungsnormen für Ziegen erstellt wurden (INRA, 1988; AFRC, 1997), gibt es in Deutschland keine detaillierten Angaben zur Ziegenfütterung. Es gelten die Normen für Schafe auf Basis der umsetzbaren Energie und des Rohproteingehaltes der Ration (DLG, 1997).

Nach HOFMANN (1988) sind die Wiederkäuer 3 Ernährungstypen zuzuordnen. In der Chronologie der evolutionären Entwicklung und in Anpassung an die Futterbedingungen werden Konzentratselektierer, Intermediärtypen und Grasfresser unterschieden. Nach dieser Einteilung gehören Schafe (selektierend) und Rinder (nicht selektierend) zu den Grasfressern und Ziegen zu den Intermediärtypen. Mit dem unterschiedlichen Futteraufnahmeverhalten sind deutliche anatomische, morphologische und physiologische Differenzen gegeben (HOFMANN, 1988). Die Milchzusammensetzung bei Ziege und Schaf weist ebenfalls beträchtliche Unterschiede auf, so dass die Bedarfsnormen für Ziege und Schaf nicht identisch sein können.

Die mikrobielle Proteinsynthese im Pansen kann bei laktierenden Hochleistungskühen nicht die für die Milchsynthese notwendige Proteinmenge am Duodenum bereitstellen. Der Einsatz von geschütztem Protein ist daher in der Milchviehfütterung eine übliche Strategie, den hohen Proteinbedarf zu decken. In der Ziegenfütterung wurden erst in den letzten Jahren vereinzelt Untersuchungen zur Nutzung von im Pansen unabbaubarem Protein mit zum Teil widersprüchlichen Ergebnissen durchgeführt (BRUNBELLUT et al., 1990; PAILAN und KAUR, 1996; MISHRA und RAI, 1996; HADJIPANAYIOTOU et al., 1996; HADJIPANAYIOTOU und PHOTIOU, 1995; TEWATIA et al., 1995; HADJIPANAYIOTOU, 1995; BORNEMANN, 1994).

Diese Mitteilung berichtet über Auswirkungen unterschiedlichen Energieangebotes für die Milchproduktion (entsprechend deutscher und internationaler Bedarfsangaben) und den Einfluss des Proteingehaltes in der Ration und der Abbaubarkeit des Proteins im Pansen auf die Milchleistung der Bunten Deutschen Edelziege.

\section{Material und Methodik}

\section{Versuchsablauf}

In 2 aufeinanderfolgenden Jahren (2000 und 2001) wurden Fütterungsversuche während der ersten 150 Laktationstage durchgeführt. Zu jedem Versuch erfolgte eine Unterteilung der Tiere in 2 Hauptgruppen, die entweder mit einem hohen $(\mathrm{H})$ oder niedrigen (N) Energieangebot pro Liter Milch versorgt wurden. Die angebotene Energie entsprach annähernd den Bedarfsangaben der GfE (DLG, 1997) (H) bzw. dem Mittel verschiedener internationaler Normen (N) (AFRC, 1997; INRA, 1988) und betrug 7,2 MJ 
ME/L (H) bzw. 5,3 MJ ME/L (N) im ersten Versuch (V1, 2000) und 6,4 MJ ME/L (H) bzw. 5,4 MJ ME/L (N) im zweiten Versuch (V2, 2001).

Die Hauptgruppen wurden weiter unterteilt in die Rationsgruppen geschütztes (Formaldehyd behandeltes, P) oder ungeschütztes (U) Sojaextraktionsschrot (SES), so dass sich, bei ausgeglichenem Proteingehalt der Rationen je Versuch der Anteil des im Pansen abbaubaren Proteins (RDP) um 13\% (V1) bzw. 30\% (V2) unterschied. Der Proteingehalt in den Rationen war niedrig 12,0-12,3\% in der TS (V1) bzw. hoch 15,9 $16,6 \%$ der TS (V2).

\section{Haltung und Fütterung}

Insgesamt standen für die Versuche 45 Ziegen der Rasse Bunte Deutsche Edelziege der Versuchsstation des Institutes zur Verfügung.

Die Ziegen waren jeweils im September des Vorjahres angepaart worden. Sie wurden anschließend in einem offenen Gruppenstall mit Auslauf untergebracht. Die Ration bestand aus Heu (ad libitum) und einem im Verlauf der Trächtigkeit steigenden Konzentratangebot (bis 600g Pellets $+100 \mathrm{~g}$ Trockenschnitzel/Tag). Die handelsüblichen Pellets für Schafe (Typ 047) enthielten 5,9 MJ NEL und 160 g Rohprotein /kg TS sowie Mineralstoffe.

2 Wochen vor dem Ablammen wurden die Tiere im Versuchsstall mit Einzelboxen (1,5 x 2m) und Stroheinstreu untergebracht. Nach dem Ablammen verblieben die Ziegenlämmer für 48 Stunden bei den Müttern.

Die Boxen waren so ausgestattet, dass Sicht- und Berührungskontakt möglich waren. Wasser stand ad libitum zu Verfügung. In dieser Gewöhnungsperiode wurde den Ziegen Urso Vitamin D3000 (2x10 i.E. Cholecalziferol) i.m. appliziert.

Die Ration bestand aus Wiesenheu (ad libitum, Mitte bis Ende der Blüte) und Pellets, die stufenweise anstelle des ursprünglichen Konzentratangebotes eingesetzt wurden. Die Versuchspellets bestanden aus Trockenschnitzeln, Gerste und Sojaextraktionsschrot (SES) im Verhältnis 10:4:1 (V1) bzw. aus Trockenschnitzeln, Gerste, SES, Weizenkleie und Melasseschnitzeln im Verhältnis 3:4:1,4:1:0,3 (V2). Die Versorgung mit Ca und P wurde entsprechend dem Bedarf (NRC, 1981) über Mineralstoffe für laktierende Schafe gesichert.

Der Nährstoffgehalt der Ration war so kalkuliert, dass ein vollständiger Verzehr der angebotenen Ration möglich war. Die unterschiedlichen Energieniveaus wurden durch die Höhe des Futterangebotes eingestellt.

Die Futtermengen wurden wöchentlich nach der individuellen Milchleistung und der Körpermasseentwicklung für jedes Tier berechnet. Dabei wurden in der ersten Laktationsphase (1.-5. Woche) die Futtermengen entsprechend der erwarteten Milchmengenleistung für $0,5 \mathrm{~kg}$ Milch vorgehalten.

Die Zusammensetzung der Futterkomponenten ist in Tabelle 1 dargestellt. Als Größenordnung des Rohproteins wurde umsetzbares Protein (MP) und für die Energie die umsetzbare Energie ME entsprechend AFRC (1997) angegeben.

Gefüttert wurde 2 mal täglich mit etwa gleich großen Rationsteilen jeweils nach dem Melken (6.00 und 16.00 Uhr). Der Futterverzehr wurde täglich erfasst. Wöchentlich erfolgte an 3 aufeinanderfolgenden Tagen die Erfassung der Milchmenge. 14tägig wurden die Ziegen gewogen. 
Die realisierte Tierzahl, durchschnittliche LM der Versuchstiere (nicht tragend), die Laktationsnummer und die durchschnittliche Milchleistung des Vorjahres (bei höherer Laktation) je Gruppe ist in Tabelle 2 aufgezeigt.

Tabelle 1

Zusammensetzung der Rationsbestandteile (g/kg TS) und berechnete Energiegehalte (MJ ME/kg TS) (Nutrient contents of components (g/kg DM) and calculated energy contents (MJ ME/kg DM))

\begin{tabular}{|c|c|c|c|c|c|}
\hline & Trockensubstanz (TS) & $\begin{array}{l}\text { Rohprotein } \\
\text { (XP) }\end{array}$ & $\begin{array}{l}\text { Umsetzbares } \\
\text { Protein (MP) }\end{array}$ & $\begin{array}{l}\text { Rohfaser } \\
\text { (XF) }\end{array}$ & $\begin{array}{c}\text { Energiegehalt } \\
\text { MJME }\end{array}$ \\
\hline & \multicolumn{5}{|c|}{ Versuch V1 (niedrige Proteinversorgung) } \\
\hline Heu & 860 & 113 & 79 & 283 & 9,7 \\
\hline Pellets (U) & 900 & 124 & 114 & 129 & 12,1 \\
\hline \multirow[t]{2}{*}{ Pellets (P) } & 900 & 129 & 123 & 126 & 12,1 \\
\hline & \multicolumn{5}{|c|}{ Versuch V2 (hohe Proteinversorgung) } \\
\hline Heu & 912 & 114 & 79 & 300 & 9,7 \\
\hline Pellets (U) & 886 & 178 & 125 & 92 & 12,1 \\
\hline Pellets (P) & 887 & 180 & 142 & 95 & 12,1 \\
\hline
\end{tabular}

Tabelle 2

Gruppenaufteilung der Versuchstiere (Group distribution of animals)

\begin{tabular}{|c|c|c|c|c|c|c|c|c|}
\hline \multirow{3}{*}{$\begin{array}{r}\text { Fütterungsniveau } \\
\text { Soja }\end{array}$} & \multicolumn{4}{|c|}{ niedrige Proteinversorgung (V1) } & \multicolumn{4}{|c|}{ hohe Proteinversorgung (V2) } \\
\hline & \multicolumn{2}{|c|}{ hoch } & \multicolumn{2}{|c|}{ niedrig } & \multicolumn{2}{|c|}{ hoch } & \multicolumn{2}{|c|}{ niedrig } \\
\hline & $\begin{array}{l}\text { unge- } \\
\text { schützt }\end{array}$ & $\begin{array}{c}\text { ge- } \\
\text { schützt }\end{array}$ & $\begin{array}{l}\text { unge- } \\
\text { schützt }\end{array}$ & $\begin{array}{c}\text { ge- } \\
\text { schützt }\end{array}$ & $\begin{array}{l}\text { unge- } \\
\text { schützt }\end{array}$ & $\begin{array}{c}\text { ge- } \\
\text { schützt }\end{array}$ & $\begin{array}{l}\text { unge- } \\
\text { schützt }\end{array}$ & $\begin{array}{c}\text { ge- } \\
\text { schützt }\end{array}$ \\
\hline Tierzahl gesamt & 5 & 6 & 7 & 5 & 5 & 5 & 6 & 6 \\
\hline $\begin{array}{l}\text { davon Tiere in } \\
\text { erster Laktation }\end{array}$ & 3 & 4 & 4 & 2 & 1 & 1 & 1 & 1 \\
\hline weitere Laktationen & 2 & 2 & 3 & 3 & 4 & 4 & 5 & 5 \\
\hline $\begin{array}{l}\varnothing \mathrm{LM} / \mathrm{kg} \text { (nicht } \\
\text { tragend)* }\end{array}$ & $47,8 \pm 7,8$ & $41,9 \pm 9,4$ & $47,2 \pm 8,9$ & $53,2 \pm 9,5$ & $46,3 \pm 4,9$ & $47,9 \pm 10,9$ & $49,6 \pm 9,4$ & $50,2 \pm 9,6$ \\
\hline Ø Trächtigkeitsnummer & $1,6 \pm 0,9$ & $1,7 \pm 1,0$ & $1,6 \pm 0,8$ & $2,0 \pm 1,0$ & $2,2 \pm 0,4$ & $2,2 \pm 0,4$ & $2,83 \pm 0,4$ & $2,67 \pm 0,4$ \\
\hline $\begin{array}{l}\varnothing \text { Milchleistung des } \\
\text { Vorjahres/L pro } 150 \\
\text { Tage }\end{array}$ & 369 & 397 & 399 & 413 & 366 & 413 & 363 & 391 \\
\hline
\end{tabular}

\section{Analytik}

Die Futtermittel wurden entsprechend der Weender Analyse auf Rohnährstoffe analysiert (Methodenbuch III, 1997). Die Milchanalytik erfolgte im Landeskontrollverband Berlin /Brandenburg e.V. in Waldsieversdorf, für Fett-, Protein-und Laktosegehalt mittels Infrarot Spektroskopie am Analysenautomat Combi VOOSS 4500. Der Milchharnstoffgehalt wurde spektrophotometrisch mit der Diacetylmonoxim-Methode analysiert.

\section{Berechnungen}

Die Berechnung der Futterrationen erfolgte auf Basis der chemischen Zusammensetzung der Versuchspellets und der Einzelkomponenten sowie den Tabellenwerten (DLG- Futterwerttabellen für Wiederkäuer, 1997) der Einzelkomponenten (Gehalte an umsetzbarer Energie und unabbaubarem Rohprotein (UDP) bzw. im Pansen abbaubarem Protein (RDP)). Als Gehaltgröße des Proteins diente das umsetzbare Protein 
(MP), da für das, bei Milchkühen nach DLG-Tabellen (1997) übliche, am Duodenum nutzbare Protein (nXP) keine Bedarfswerte für Ziegen existieren. Der Gehalt an umsetzbarem Protein (MP) errechnet sich entsprechend AFRC (1997) als Summe aus wahrem verdaulichen Mikrobenprotein (TMP) und im Pansen nicht abbaubarem verdaulichen Protein (DUP). Dabei wurden angenommen: 9,6g Mikrobenproteinsynthese/MJ ME Aufnahme; 75\% des Mikrobenproteins sind wahres Protein; die Verdaulichkeit des Mikrobenproteins am Dünndarm beträgt 85\%. Der DUP-Gehalt wurde aus dem Anteil an UDP (Kalkulation aus den Tabellenwerten) mit einer Verdaulichkeit von $85 \%$ berechnet.

Der Kalkulation der Bedarfswerte liegen nachfolgende Überlegungen zugrunde:

- Der Erhaltungsbedarf als Mittel der bereits zitierten Bedarfsnormen bei der Berechnung der Rationen beträgt $430 \mathrm{~kJ} \mathrm{ME} / \mathrm{kg} \mathrm{W}^{0,75}$. Dabei wurde der nach neueren Erkenntnissen zu beachtende Zuschlag für die Aktivität der Tiere (AFRC, 1997) nicht berücksichtigt, da die Ziegen während des Versuches im Stall gehalten wurden.

- Der Energiebedarf für die Milchleistung errechnet sich nach AFRC (1997) auf Basis der Gleichung von TYRELL und REID (1965)

Nettoenergie MJ/kg Milch = 0,376 x \% Fett + 0,209 x \% Protein + 0,948 mit einem Wirkungsgrad der Nutzung der ME für Milch von 0,63 (SUTTON und ALDERMAN, 2000). Nach DLG Tabellen (1997) beträgt der Bedarf für $1 \mathrm{~L}$ Milch 8,0 MJ ME.

- Der Proteinbedarf für Erhaltung wird mit 3,25g RP/ kg W ${ }^{0,75}$ (DLG, 1997) bzw. 2,35 g MP /kg W ${ }^{0,75}$ (AFRC, 1997) angegeben.

- Der Bedarf an MP für die Milchproduktion wurde anhand des wahren Proteingehaltes in der Milch (0,9 x Proteingehalt) kalkuliert mit einem Wirkungsgrad der MP Ausnutzung von 0,68. Der Rohproteinbedarf je Liter Milch entsprechend der DLGTabellen (1997), umgerechnet auf den durchschnittlichen Proteingehalt der Ziegenmilch, wurde mit $90 \mathrm{~g}$ angenommen.

Für eine $50 \mathrm{~kg}$ Ziege werden für Erhaltung und die Bildung pro Liter Milch (mit etwa 3,7\% Fett und 2,9\% Protein) folgende Energie- und Proteinmengen nach den internationalen Normen benötigt:

8,4 MJ ME für Erhaltung und 4,8 MJ ME pro Liter Milch sowie 62 g Rohprotein bzw. 44,2 g MP für Erhaltung und 41g MP pro Liter Milch (berechnet aus den o.g. Bedarfsangaben, AFRC, 1997).

\section{Statistische Berechnungen}

Die Datenanalyse erfolgte mit Hilfe der Univariaten GLM-Prozedur von SPSS 9.0 bzw. 10.0 für Windows. Als Haupteinflüsse konnten das Fütterungsniveau (Hoch oder Niedrig), die Proteinqualität (geschützt $\mathrm{P}$ oder ungeschützt $\mathrm{U}$ ) die Laktationsnummer (1 oder höher $=2$ ) und die Lammzahl (1 oder 2 ) sowie im V1 der Gesundheitsstatus (1 bis 3) im benutzten statistischen Modell berücksichtigt werden. Zur Berechnung standen die wöchentlichen Mittelwerte des 21 wöchigen Versuchszeitraumes zur Verfügung.

Das Grundmodell der Varianzanalyse ist:

$$
\begin{gathered}
Y_{i j k l m}=\mu+\alpha_{i}+\beta_{j}+\gamma_{k}+\delta_{l}+\pi_{m}+\left(\alpha \beta_{i j}\right)+\left(\alpha \beta \gamma_{i j k}\right)+e_{i j k l m} \\
Y_{i j k l m}=\text { gemessener Wert für eine abhängige Variable }
\end{gathered}
$$




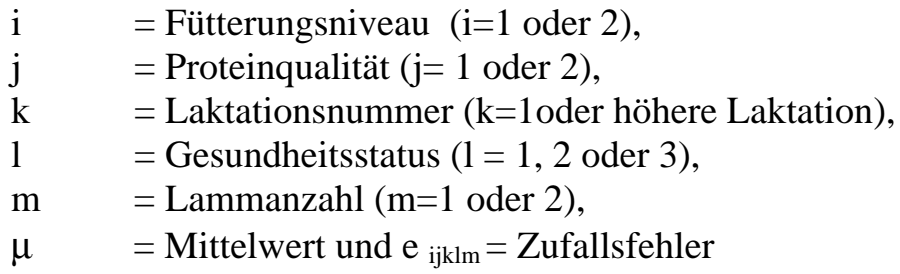

\section{Ergebnisse}

\section{Futteraufnahme}

Die Zusammensetzung der verzehrten Ration ist in Tabelle 3 dargestellt. Die Energiegehalte je $\mathrm{kg}$ TS waren mit 11,2 bis 11,6 MJ ME/kg TS ausgeglichen und entsprachen den neuesten AFRC-Anforderungen von 11,3 MJ ME/kg TS für optimale Fermentationsbedingungen im Pansen (SUTTON und ALDERMAN, 2000). Der durchschnittliche Proteingehalt betrug im 1 . Versuch 12,2\% und im 2. Versuch 16,3\% und entsprach dem geplanten niedrigen bzw. hohem Proteinangebot. Der Unterschied im Gehalt an UDP in der Ration zwischen der ungeschützten und geschützten Proteingruppe betrug im V1 $=15 \%$ und im V2 $=30 \%$. Der Gehalt an RDP lag im V1 mit 6,95 g RDP/MJ ME deutlich unter und mit 9,26 g RDP/MJ ME im V2 oberhalb der AFRCEmpfehlung von 7,81 g RDP/MJ ME zur effizienten N-Nutzung im Pansen.

Tabelle3

Zusammensetzung der aufgenommenen Rationen und kalkulierte Gehalte an Energie- und Proteinfraktion (Chemical composition of consumed diets and calculated contents of energy and the protein fractions)

\begin{tabular}{|c|c|c|c|c|c|c|c|c|}
\hline \multirow{3}{*}{$\begin{array}{r}\text { Energieniveau } \\
\text { Soja }\end{array}$} & \multicolumn{4}{|c|}{ niedrige Proteinversorgung (V1) } & \multicolumn{4}{|c|}{ hohe Proteinversorgung (V2) } \\
\hline & \multicolumn{2}{|c|}{ hoch } & \multicolumn{2}{|c|}{ niedrig } & \multicolumn{2}{|c|}{ hoch } & \multicolumn{2}{|c|}{ niedrig } \\
\hline & $\begin{array}{c}\text { unge- } \\
\text { schützt }\end{array}$ & $\begin{array}{c}\text { ge- } \\
\text { schützt }\end{array}$ & $\begin{array}{c}\text { unge- } \\
\text { schützt }\end{array}$ & $\begin{array}{c}\text { ge- } \\
\text { schützt }\end{array}$ & $\begin{array}{l}\text { unge- } \\
\text { schützt }\end{array}$ & $\begin{array}{c}\text { ge- } \\
\text { schützt }\end{array}$ & $\begin{array}{l}\text { unge- } \\
\text { schützt }\end{array}$ & $\begin{array}{c}\text { ge- } \\
\text { schützt }\end{array}$ \\
\hline $\begin{array}{l}\text { Rohprotein (XP; \% der } \\
\text { TS) }\end{array}$ & 12,32 & 12,38 & 11,92 & 12,21 & 16,4 & 16,6 & 15,9 & 16,1 \\
\hline Rohfaser (XF; \% der TS) & 16,70 & 17,27 & 19,04 & 17,98 & 13,7 & 13,8 & 15,3 & 15,4 \\
\hline RDP (\% des XP) & 65,51 & 61,07 & 68,16 & 62,00 & 70,2 & 60,3 & 70,9 & 61,4 \\
\hline UDP & 34,49 & 38,93 & 31,84 & 38,00 & 29,8 & 39,7 & 29,9 & 38,6 \\
\hline RDP (g/MJ ME) & 6,97 & 6,70 & 7,30 & 6,83 & 9,91 & 8,63 & 9,86 & 8,64 \\
\hline RDP (g/kg TS) & 80,7 & 75,6 & 81,2 & 75,7 & 115,1 & 100,0 & 112,7 & 98,9 \\
\hline MP (g/kg TS) berechnet ${ }^{1)}$ & 106,2 & 111,1 & 100,7 & 108,9 & 114,0 & 128,5 & 110,3 & 124,5 \\
\hline $\begin{array}{l}\text { Energie (MJ ME/kg TS) } \\
\text { berechnet }^{2)}\end{array}$ & 11,58 & 11,41 & 11,19 & 11,32 & 11,57 & 11,60 & 11,38 & 11,41 \\
\hline MP:ME & 9,17 & 9,73 & 9,00 & 9,62 & 9,85 & 11,08 & 9,69 & 10,91 \\
\hline
\end{tabular}

Der Rohfasergehalt lag im Mittel bei 17,8\% (V1) bzw. 14,6 \% (V2).

Die Futteraufnahme in g TS pro Tier und Tag pro kg metabolische LM schwankte zwischen 69-172 g/Tag und lag innerhalb der für Ziegen im gemäßigten Klima angegebenen Schwankungsbreite von 47-181 g (SAUVANT et al., 1991). Für eine 50 kg Ziege entspricht die durchschnittliche Futteraufnahme einer Menge von 2,2 kg TS pro Tag, das sind 4,4\% der Lebendmasse. 
Tabelle 4

Einfluss des Energieniveaus auf die tägliche Futter- und Nährstoffaufnahme bezogen auf die metabolische Lebendmasse $\left(\mathrm{kg} \mathrm{W}^{0,75}\right.$ ) (Influence of energy level on daily feed- and nutrient intake based on metabolic body weight $\left.\left(\mathrm{kg} \mathrm{W}^{0,75}\right)\right)$

\begin{tabular}{|c|c|c|c|c|c|c|}
\hline & \multicolumn{3}{|c|}{ niedrige Proteinversorgung } & \multicolumn{3}{|c|}{ hohe Proteinversorgung } \\
\hline & \multicolumn{2}{|c|}{ Energieniveau } & \multirow{2}{*}{$\begin{array}{l}\text { Signi- } \\
\text { fikanz }\end{array}$} & \multicolumn{2}{|c|}{ Energieniveau } & \multirow{2}{*}{$\begin{array}{l}\text { Signi- } \\
\text { fikanz }\end{array}$} \\
\hline & Hoch & Niedrig & & Hoch & Niedrig & \\
\hline Heu TS-Aufnahme (g/Tag) & $26 \pm 0,45$ & $34 \pm 0,52$ & $\mathrm{xxx}$ & $31 \pm 0,52$ & $36 \pm 0,46$ & $\mathrm{xxx}$ \\
\hline Pellets TS-Aufnahme (g/Tag) & $86 \pm 1,33$ & $66 \pm 1,52$ & $\mathrm{xxx}$ & $104 \pm 1,86$ & $84 \pm 1,64$ & $\mathrm{xxx}$ \\
\hline TS-Aufnahme gesamt (g/Tag) & $112 \pm 1,36$ & $100 \pm 1,55$ & $\mathrm{xxx}$ & $135 \pm 1,75$ & $120 \pm 1,55$ & $\mathrm{xxx}$ \\
\hline XP-Aufnahme(g/Tag) & $13,8 \pm 0,18$ & $12,1 \pm 0,2$ & $\mathrm{xxx}$ & $22,1 \pm 0,32$ & $19,1 \pm 0,28$ & $\mathrm{xxx}$ \\
\hline MP-Aufnahme (g/Tag) & $12,2 \pm 0,16$ & $10,5 \pm 0,18$ & $\mathrm{xxx}$ & $16,4 \pm 0,24$ & $14,0 \pm 0,21$ & $\mathrm{xxx}$ \\
\hline ME-Aufnahme (MJ/Tag) & $1,29 \pm 0,02$ & $1,12 \pm 0,02$ & $\mathrm{XXX}$ & $1,56 \pm 0,02$ & $1,36 \pm 0,02$ & $\mathrm{xxx}$ \\
\hline
\end{tabular}

Tabelle 4 zeigt die Daten der Futteraufnahme in Abhängigkeit vom Energieniveau. Da die unterschiedlichen Fütterungsniveaus durch die angebotene Pelletmenge eingestellt wurden, war das hohe Energieniveau erwartungsgemäß mit einer höheren Futteraufnahme verbunden. Im Vergleich der beiden Versuche war sie im V2 trotz identischen Angebotes zum Beginn der Versuche deutlich höher als im ersten Versuch.

Das geringere Pelletangebot in den N-Gruppen wurde von den Ziegen durch eine erhöhte Heuaufnahme teilweise kompensiert.

Die aufgenommenen Mengen an umsetzbarer Energie überschreiten den Erhaltungsbedarf um das 2,1 bis 3,2 fache. Das entspricht einer möglichen Milchleistung von 2,1 bis $4,2 \mathrm{~L} / \mathrm{Tag}$.

Tabelle 5

Einfluss der Proteinart (geschützt/ungeschützt) auf die tägliche Futter- und Nährstoffaufnahme bezogen auf die metabolische Lebendmasse $\left(\mathrm{kg} \mathrm{W}^{0,75}\right)$ (Influence of protein (protected, non protected) on daily feed- and nutrient intake based on metabolic body weight $\left.\left(\mathrm{kg} \mathrm{W}^{0,75}\right)\right)$

\begin{tabular}{|c|c|c|c|c|c|c|}
\hline \multirow[b]{2}{*}{ Parameter } & \multicolumn{3}{|c|}{ niedrige Proteinversorgung } & \multicolumn{3}{|c|}{ hohe Proteinversorgung } \\
\hline & $\begin{array}{c}\text { Soja } \\
\text { ungeschützt }\end{array}$ & $\begin{array}{c}\text { Soja } \\
\text { geschützt }\end{array}$ & $\begin{array}{l}\text { Signi- } \\
\text { fikanz }\end{array}$ & $\begin{array}{c}\text { Soja } \\
\text { ungeschützt }\end{array}$ & $\begin{array}{c}\text { Soja } \\
\text { geschützt }\end{array}$ & $\begin{array}{l}\text { Signi- } \\
\text { fikanz }\end{array}$ \\
\hline Heu TS-Aufnahme (g/Tag) & $29 \pm 0,71$ & $31 \pm 0,31$ & NS & $34 \pm 0,46$ & $33 \pm 0,52$ & NS \\
\hline Pellets TS-Aufnahme (g/Tag) & $76 \pm 1,29$ & $76 \pm 1,59$ & NS & $88 \pm 1,65$ & $100 \pm 1,86$ & $\mathrm{xxx}$ \\
\hline TS-Aufnahme gesamt (g/Tag) & $105 \pm 1,32$ & $107 \pm 1,62$ & NS & $122 \pm 1,56$ & $133 \pm 1,76$ & $\mathrm{xxx}$ \\
\hline XP Aufnahme(g/Tag) & $12,7 \pm 0,17$ & $13,2 \pm 0,21$ & $\mathrm{xxx}$ & $19,6 \pm 0,28$ & $21,7 \pm 0,32$ & $\mathrm{xxx}$ \\
\hline MP Aufnahme (g/Tag) & $10,9 \pm 0,16$ & $11,7 \pm 0,19$ & $\mathrm{xxx}$ & $13,7 \pm 0,21$ & $16,7 \pm 0,24$ & $\mathrm{xxx}$ \\
\hline ME Aufnahme (MJ/Tag) & $1,20 \pm 0,02$ & $1,21 \pm 0,02$ & NS & $1,40 \pm 0,02$ & $1,52 \pm 0,02$ & $\mathrm{xxx}$ \\
\hline
\end{tabular}

signifikante Differenz xxx $P<0.001$; NS = nicht signifikant

Der Einfluss der Proteinqualität auf die Futteraufnahme ist in Tabelle 5 aufgezeigt. Bei einem niedrigen Proteinangebot (V1) führt die Erhöhung des UDP-Anteils nicht zur Veränderung der Futteraufnahme. Im Versuch V2 mit hohem Proteinangebot wirkte sich die Verfütterung von geschütztem Soja positiv auf die Futteraufnahme aus. 
In keinem Fall wurde die in der Literatur teilweise beschriebene Senkung der Futteraufnahme durch geschütztes Sojaprotein (HADJIPANAYIOTOU und MORANDFEHR, 1991; BRUN-BELLUT et al., 1990) gefunden.

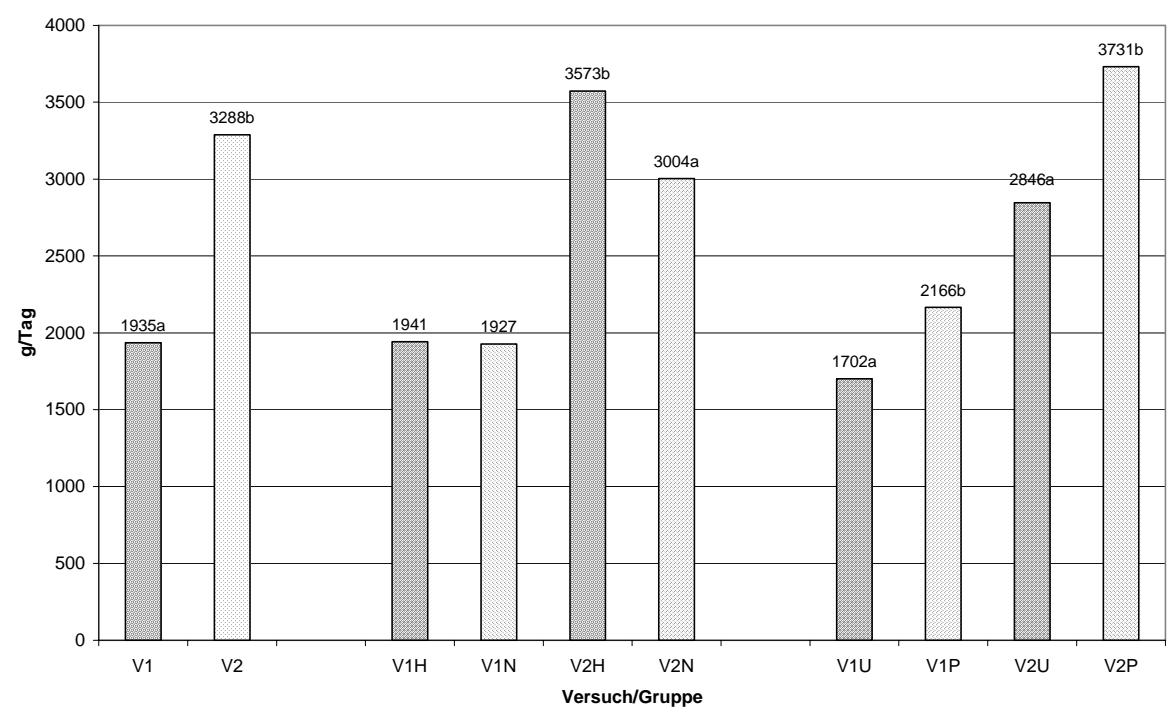

Abb. 1: Milchleistung (FCM) in Abhängigkeit vom Protein- und Energieniveau sowie vom Anteil an UDP in der Ration (Milk yield (FCM) in relation to protein- and energy level and UDP level (protein protection) in the diet)

Unterschiedliche Buchstaben innerhalb eines Versuches = Signifikante Unterschiede

$\mathrm{H}=$ hohes Energieniveau der Ration; $\mathrm{N}=$ niedriges Energieniveau der Ration

$\mathrm{P}=$ Einsatz von geschütztem Sojaextraktionsschrot; $\mathrm{U}=$ Einsatz von ungeschütztem Sojaextraktionsschrot

\section{Milchleistung}

Die Milchleistung in beiden Versuchen in Abhängigkeit vom Energieniveau und der Art des Proteins ist in Abbildung 1 dargestellt. Bei gleichem Energieangebot und ausgeglichener Energiekonzentration (MJ ME/kg TS) in den Versuchen V1 und V2 zeigt sich eine deutliche Steigerung der Milchmenge auf $170 \%$ im Versuch V2.

Trotz höherer Energieaufnahme (+15\%) der Gruppe H im V1 war die Milchmenge nicht höher als in der N-Gruppe. Im 2. Versuch ergab sich eine signifikant höhere Milchleistung bei gesteigerter ME-Aufnahme.

In beiden Versuchen führte die Erhöhung des UDP-Anteils zur Steigerung der Milchmenge (FCM) um 27 bzw. 31\%.

Die Zusammensetzung der Milchinhaltsstoffe (Tab. 6) zeigte insgesamt mit 3,45\% Fett, 2,9\% Protein und 4,44\% Laktose der Literatur entsprechende Werte für die Rasse Bunte Deutsche Edelziege. Die Bunte Deutsche Edelziege gehört zu den Rassen, die einen vergleichbar niedrigen Nettoenergiegehalt der Milch aufweisen. Im vorliegenden Versuch betrug er im Mittel 2,81 MJ/kg mit einer Variationsbreite von 2,49 bis 3,06 MJ.

Die produzierte Milchmenge je MJ ME Aufnahme zeigt keine gerichtete Tendenz in Abhängigkeit zum Fütterungsniveau (Tab. 6).

\section{Diskussion}

Die Höhe des Futterangebotes richtete sich nach der Milchleistung und der benötigten Energie für den Erhaltungsbedarf und war zu Beginn der Laktation für beide Versuche in den entsprechenden Gruppen jeweils gleich. Trotzdem verzehrten die Tiere im V2 
Tabelle 6

Einfluss der Energie und des Protein-Gehaltes der Ration auf die tägliche Milchleistung FCM, Milchinhaltsstoffe, berechnete Milchenergie und tägliche Zunahme (g/Tag)

(Effect of energy- and protein content of diet on daily FCM yield (g/d), milk nutrient (\%) and urea (mg/L) content, calculated milk energy (MJ/kg) and daily body weight change $(\mathrm{g} / \mathrm{d}))$

\begin{tabular}{|c|c|c|c|c|c|c|c|c|c|c|}
\hline \multirow{4}{*}{$\begin{array}{r}\text { Parameter } \\
\\
\text { Energieniveau } \\
\text { Soja } \\
\end{array}$} & \multicolumn{5}{|c|}{ Behandlungskombinationen } & \multicolumn{5}{|c|}{ Behandlungskombinationen } \\
\hline & \multicolumn{5}{|c|}{ niedrige Proteinversorgung } & \multicolumn{5}{|c|}{ hohe Proteinversorgung } \\
\hline & & & & & & & & nie & & \\
\hline & ungeschützt & geschützt & ungeschützt & geschützt & Signifikanz & ungeschützt & geschützt & ungeschützt & geschützt & Signifikanz \\
\hline FCM g/Tag & $1806 a \pm 73$ & $2078 b \pm 70,9$ & $1600 \mathrm{~d} \pm 67$ & $2254 a \pm 91,1$ & $\mathrm{xx}$ & $2953 a \pm 139,3$ & $4194 c \pm 139,3$ & $2739 a \pm 128,2$ & $3268 b \pm 138,1$ & $\mathrm{xxx}$ \\
\hline Milch-Fett (\%) & $2,84 c \pm 0,08$ & $3,43 \mathrm{~b} \pm 0,08$ & $3,48 b \pm 0,08$ & $3,60 a \pm 0,10$ & $\mathrm{xx}$ & $3,17 a \pm 0,11$ & $4,03 c \pm 0,11$ & $3,59 b \pm 0,10$ & $3,61 b \pm 0,11$ & $\mathrm{xxx}$ \\
\hline Milch-Protein (\%) & $2,82 c \pm 0,05$ & $3,05 a \pm 0,05$ & $3,12 \mathrm{a} \pm 0,04$ & $2,96 b \pm 0,06$ & $\mathrm{xxx}$ & $2,70 a \pm 0,05$ & $2,86 b \pm 0,05$ & $2,87 \mathrm{~b} \pm 0,05$ & $2,81 \mathrm{ab} \pm 0,05$ & $\mathrm{x}$ \\
\hline Milch-Lactose (\%) & $4,45 \mathrm{~d} \pm 0,02$ & $4,65 a \pm 0,02$ & $4,49 c \pm 0,02$ & $4,54 \mathrm{~b} \pm 0,03$ & $\mathrm{xxx}$ & $4,54 a \pm 0,02$ & $4,64 \mathrm{~b} \pm 0,02$ & $4,54 a \pm 0,02$ & $4,54 a \pm 0,02$ & $\mathrm{xx}$ \\
\hline $\begin{array}{l}\text { Milch-Harnstoff } \\
\text { (mg/l) }\end{array}$ & $175 \pm 6,9$ & $183 \pm 6,7$ & $192 \pm 6,4$ & $204 \pm 8,7$ & N.S. & $406 a \pm 10,78$ & $451 b \pm 10,78$ & $431 b \pm 9,93$ & $427 b \pm 10,69$ & $\mathrm{x}$ \\
\hline Milch NE (MJ/kg) & $2,49 b \pm 0,041$ & $2,81 a \pm 0,04$ & $2,81 \mathrm{a} \pm 0,04$ & $2,83 a \pm 0,05$ & $\mathrm{xxx}$ & $2,70 a \pm 0,05$ & $3,06 c \pm 0,05$ & $2,90 \mathrm{~b} \pm 0,05$ & $2,90 \mathrm{~b} \pm 0,05$ & $\mathrm{xxx}$ \\
\hline $\begin{array}{l}\text { FCM (g/MJ ME) } \\
\text { Aufnahme }\end{array}$ & $68 \pm 2,98$ & $92 \pm 2,89$ & $76 \pm 2,73$ & $95 \pm 3,71$ & N.S. & $95 a \pm 4,5$ & $125 c \pm 4,5$ & $112 \mathrm{~b} \pm 4,1$ & $116 b c \pm 4,4$ & $*$ \\
\hline $\begin{array}{l}\text { FCM (g/g MP) } \\
\text { Aufnahme }\end{array}$ & $7,48 \pm 0,322$ & $9,42 \pm 0,31$ & $8,45 \pm 0,30$ & $9,91 \pm 0,40$ & N.S. & $9,6 a \pm 0,4$ & $11,3 \mathrm{~b} \pm 0,4$ & $11,5 b \pm 0,4$ & $10,7 b \pm 0,4$ & $*$ \\
\hline $\begin{array}{l}\text { Effizienz der Ener- } \\
\text { gie für Milchbildung } \\
\text { (MJ/MJ ME Aufn.)+ }\end{array}$ & $0,39 a \pm 0,008$ & $0,45 b \pm 0,01$ & $0,43 b \pm 0,01$ & $0,46 b \pm 0,01$ & $\mathrm{x}$ & $0,41 \mathrm{a} \pm 0,07$ & $0,44 \mathrm{~b} \pm 0,07$ & $0,44 \mathrm{~b} \pm 0,06$ & $0,44 \mathrm{~b} \pm 0,07$ & $*$ \\
\hline $\begin{array}{l}\text { Tägliche Zunahme } \\
\text { (g/Tag) }\end{array}$ & $41 \pm 16,0$ & $6 \pm 15,2$ & $17 \pm 13,4$ & $19 \pm 16,0$ & N.S. & $58 \pm 25,6$ & $14 \pm 25,6$ & $18 \pm 23,6$ & $10 \pm 25,4$ & N.S. \\
\hline
\end{tabular}

Unterschiedliche Buchstaben in einer Zeile innerhalb eines Versuches = signifikante Differenz $\mathrm{x} P<0.05$, xx $P<0.01$, xxx $P<0.001$; NS = nicht signifikant.

FCM $=4 \%$ Fett; + = Berechnet als: ME Milch/ (ME Milch + ME oberhalb Erhalt) 
deutlich mehr als im ersten Versuch. Hauptursache dafür könnte der höhere Proteingehalt der Ration V2 sein. Auf den positiven Zusammenhang zwischen Proteingehalt und Futteraufnahme bei Ziegen wiesen auch BADAMA und SUTTON (1987), BADAMA et al. (1990), MISHRA und RAI (1996) und PAILAN und KAUR (1996) hin. Die Grenze der Steigerung der Futteraufnahme für Ziegen wird bei etwa 15\% Rohprotein in der Ration angegeben (in AFRC, 1997, pp 821).

Ob auch der Proteinschutz einen Einfluss auf die Futteraufnahme hat, konnte nicht eindeutig geklärt werden. Allerdings ergab sich in keinem Fall eine geringere Futteraufnahme. Eine negative Beeinflussung des Geschmacks durch die Behandlung des SES kann jedoch in diesen Versuchen ausgeschlossen werden, da das Kraftfutter in pelletierter Form verabreicht wurde. QUICK et al. (1986) berichteten über die besondere Präferenz von Ziegen für Pellets.

Weiterhin ist im Zusammenhang mit der Fütterung von geschütztem Protein zu beachten, dass mit Erhöhung des UDP das N-Angebot im Pansen reduziert wird. Bei geringerem Proteingehalt in der Ration führt das zu einem Stickstoffmangel im Pansen. Die dadurch hervorgerufene Beeinträchtigung der Pansenfermentation ist mit einer Reduzierung der Futteraufnahme verbunden, wie BRUN-BELLUT et al. (1990) bei einem Rohproteingehalt in der Ration von 11,7\% nachwiesen. Bei einem RPGehalt von 14,7\% in der Ration hatte ein unterschiedlicher RDP-Gehalt keinen Einfluss auf die Futteraufnahme.

Im Versuch von BRUN-BELLUT et al. (1990) lag der RDP-Anteil in der mit geschütztem Sojaprotein gefütterten Gruppe um $29 \%$ niedriger als in der Kontrollgruppe.

In vorliegend beschriebenen Versuchen betrug der Unterschied im RDP-Gehalt zwischen den Gruppen U und P 7,9\% (V1) bzw. 13,7\% (V2). Da der RDP-Bedarf pro MJ ME im ersten Versuch von allen Versuchsgruppen unterschritten und im zweiten Versuch überschritten wurde, konnte dieser Zusammenhang nicht eindeutig nachgewiesen werden.

Die höhere Energie- und Proteinaufnahme im Versuch V2 führte zu einer Steigerung der Milchleistung um 70\%. Die Aufnahme an umsetzbarer Energie erhöhte sich um 15\%. Der Verzehr an MP war um 34\% gesteigert.

Nach HADJIPANAYIOTOU und MORAND-FEHR (1991) beeinflusst die Energieaufnahme die Milchleistung am stärksten. Eine Verringerung des Energieangebotes auf 85\% des Bedarfs für die Leistung führte innerhalb von 24- 48 Stunden zur deutlichen Reduktion der Milchleistung (MORAND-FEHR und SAUVANT, 1978).

Jedoch ist mit der um 15\% erhöhten Energieaufnahme allein nicht die Milchleistungssteigerung um $70 \%$ zu erklären. Zusätzlich zur geringeren Energieaufnahme im V1 ist die wesentlich stärker reduzierte Proteinaufnahme zu berücksichtigen. Im Versuch V1 (Proteingehalt 11,9 - 12,4\%) muß davon ausgegangen werden, dass ein Mangel an RDP in allen Gruppen vorlag. Die RDP-Konzentrationen lagen im V1 zwischen 7,6 und 8,1\% der TS (Tab. 3). Die damit verbundene, zu erwartende verringerte $\mathrm{NH}_{3}$ Konzentration im Pansen könnte zur Reduzierung der Pansenmikrobensynthese geführt haben.

Nach BRUN-BELLUT et al. (1990) sinkt die Pansenmikrobensynthese bei einem Gehalt an RDP unter 8,2 \% der TS. In diesem Falle ist auch durch N-Rezyklierung ein NMangel nicht mehr ausreichend auszugleichen. 
Bei der Rationsgestaltung ist grundsätzlich das gebotene Verhältnis von langsam und schnell verfügbarer Energie und dem im Pansen verfügbaren Protein zu berücksichtigen.

Im vorliegenden Versuch (V1) stand dem hohen Anteil an schnell verfügbarer Energie aus Gerste und Trockenschnitzeln ein zu geringer RDP-Gehalt gegenüber, so dass davon auszugehen ist, dass die Effizienz der Energieverwertung für die Milchsynthese reduziert war. Auch bei dem hohen Proteinangebot im zweiten Versuch könnte der schnell verfügbare Proteinanteil im Verhältnis zur bereitgestellten schnell verfügbaren Energie noch zu gering gewesen sein. Dies wird besonders in den H-Gruppen (Tab.6) deutlich.

Die Effizienz der Nutzung der ME zur Milchsynthese liegt mit durchschnittlich 0,43 deutlich unter den in der Literatur beschriebenen Angaben von 0,63. Mit einer Energiekonzentration von 11,3 MJ ME/kg TS entsprach die Ration den Forderungen von SUTTON und ALDERMAN (2000), lag aber deutlich über den von MORAND-FEHR und SAUVANT (1978) angegebenen Werten von $10 \mathrm{MJ} \mathrm{ME} / \mathrm{kg}$ TS im 3. Laktationsmonat. Die Autoren fanden bei höherer Energiekonzentration eine Senkung der Milchleistung. Das steht im Widerspruch zu Untersuchungen an Kühen und sollte Gegenstand weiterer Untersuchungen sein.

Die Erhöhung des UDP-Anteils in der Ration durch Verabreichung von geschütztem Sojaextraktionsschrot führte in beiden Versuchen zur Steigerung der Milchleistung.

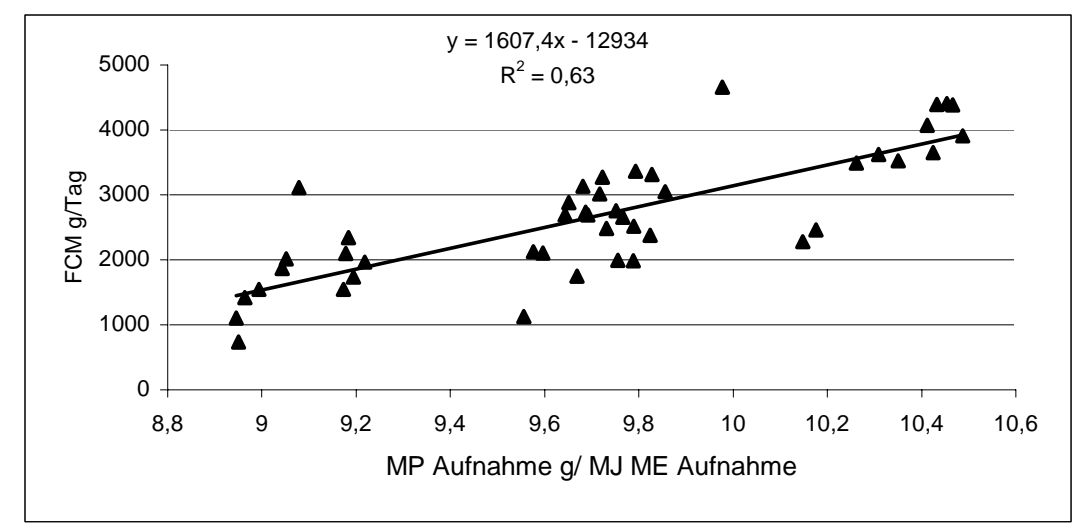

Abb. 2: Zusammenhang zwischen der Milchleistung (FCM g/Tag) und dem kalkulierten Verhältnis MP:ME Aufnahme (Relationship between FCM yield and the calculated ratio of MP:ME intake)

Mit der Steigerung des Anteils an UDP erhöhte sich die am Duodenum zur Verfügung stehende MP-Menge. Damit verbunden vergrößerte sich das Verhältnis von MP:ME, was zu einer signifikanten Erhöhung der Milchsynthese führte (Abb. 2). Auf diesen Zusammenhang wiesen bereits OLDHAM (1984) und PAULICKS (1985) hin.

Die Verabreichung von geschütztem Protein kann zu einer Steigerung der Milchleistung bei Ziegen führen, wie HADJIPANAYIOTOU und MORAND-FEHR (1991) bestätigten.

In anderen in der Literatur beschriebenen Untersuchungen konnte keine Steigerung der Milchleistung durch einen erhöhten UDP-Anteil erreicht werden. Die Ursachen für unterschiedliche Wirkungen des Proteinschutzes können nach SCHONGOETHE (1996) vielfältig sein: Mangel an RDP bei unveränderter Proteinaufnahme, zu starke Behandlung des Proteins, so dass es auch im Jejunum nur schwer verdaulich ist, geringe Qualität des UDP hinsichtlich der Aminosäurenzusammensetzung und Mangel an limitierenden Aminosäuren des Proteins am Duodenum. 
Der Proteingehalt der Milch kann durch die Fütterung nur begrenzt beeinflußt werden. Differenzen im Rohproteingehalt der Ration beeinflussen stärker die Milchmenge als den Proteingehalt. Jedoch kann die Erhöhung des Anteils an UDP mit hoher Proteinqualität eine Steigerung des Milchproteingehaltes bewirken (DE PETERS und CANT, 1992; BRODERICK et al., 1974; SCHINGOETHE et al., 1988). Auch wir fanden eine Steigerung des Milchproteingehaltes bei Verabreichung von geschütztem Protein (Tab. 5).

Die unterschiedlichen Fettgehalte in den Versuchsgruppen (Tab. 6) könnten im Wesentlichen durch eine erhöhte Faseraufnahme in den N-Gruppen bedingt sein.

Der Harnstoffgehalt in der Milch ist eng korreliert mit der Blutharnstoffkonzentration und diese ist ein Indikator für die N-Versorgung des Organismus. Aus Untersuchungen von BRUN-BELLUT et al. (1990) entwickelten BELLOF und WEPPERT (1996) ein 9-Felder-Schema zur Beurteilung der Fütterungssituation der Milchziegen. Neben dem Harnstoffgehalt der Milch wird der Milchproteingehalt berücksichtigt. Danach ist eine optimale Versorgung der Ziegen (ähnliche normale Milchgehaltswerte wie Bunte Deutsche Edelziege) bei einem Proteingehalt zwischen 2,9 und 3,4 \% und einer Harnstoffkonzentration in der Milch von 200-400 mg/L gegeben. Geringere Harnstoffwerte zeigen Proteinmangel an und niedrigere Proteingehalte weisen auf Energiemangel hin. Die Harnstoffgehalte im ersten Versuch liegen zwischen 175 und 204 mg /L und sind ein Indikator für eine Grenzsituation zum Proteinmangel. Im V2 zeigt die hohe Milchharnstoffkonzentration einen Proteinüberschuß an.

Die Harnstoff- und Proteingehalte der Milch sind im Verlauf der Laktation sehr unterschiedlich. Ab der 13. Laktationswoche war eine stark erhöhte Harnstoffkonzentration (450 - 498 mg/L) in der Milch zu verzeichnen, so dass ein bedeutender Proteinüberschuss erst in diesem Laktationsstadium vorlag.

Aus den vorliegenden Ergebnissen lassen sich folgende Schlussfolgerungen ziehen:

- Eine hohe Milchleistung kann nur erzielt werden, wenn das Energie- und Proteinangebot entsprechend dem Bedarf gedeckt wird und die mikrobile Aktivität im Pansen optimal gesichert ist.

- Der Energiebedarf für die Milchbildung sollte aus der Milchzusammensetzung berechnet werden.

- Das Verhältnis zwischen MP- und ME-Aufnahme zeigt eine signifikante Korrelation zur Milchleistung.

- Das Proteinangebot sollte so gestaltet werden, dass der RDP-Anteil von 8,5 \% der TS der Ration und die RDP-Konzentration von 7,8 g/MJ ME nicht unterschritten werden. Die verfütterte Rohproteinkonzentration der Ration sollte über $12 \%$ und deutlich unter 16\% der TS liegen, wie aus den Analysen der Milchharnstoffkonzentration zu ersehen ist.

- Die Erhöhung des UDP-Anteils steigert bei gleichem Proteinangebot das Verhältnis von MP:ME und führte unter allen Fütterungsbedingungen zur Steigerung der Milchleistung. Bei ausreichender N-Versorgung des Pansens wurde durch UDPGehalte bis zu 40\% des Proteins eine Milchleistungssteigerung erzielt.

- Eine Phasenfütterung, die dem sich veränderndem Bedarf während der Laktation entspricht, scheint auch für Ziegen sinnvoll. 
AFRC:

\section{Literatur}

Technical Commitee on Responses to Nutrients. Report No. 10, The nutrition of goats CAB International, Ser. B, 67 (1997), 765-830

BADAMA, M.S.; SUTTON, J.D.:

The effect of level of protein in the concentrateson hay intake, milk production and digestive processes by Saanen goats. Animal Production, 44 (1987), 496

BADAMA, M.S.; SUTTON, J.D.; OLDHAM, J.D.; MOWLEM, A.:

The effect of amount of protein in the concentrates on hay intake and rate of passage, diet digestibility and milk production in British Saanen goats. Animal Production, 51 (1990), 333-342

BELLOF, G.: WEPPERT, M.:

Die Beurteilung der Energie- und Eiweißversorgung bei der Milchziege mit Hilfe von Milchharnstoff und Milcheiweißgehalt. (Predicting energy and protein supply status of dairy goat from urea and protein of milk content). Der Ziegenzüchter 6 (1996), 2-12

BORNEMANN, S.:

Auswirkungen einer reduzierten Rohproteinversorgung auf die N-Ausscheidung und die intermediäre Umsetzung von ${ }^{15} \mathrm{~N}$-markiertem Harnstoff bei laktierenden Ziegen. Rheinische Friedrich-WilhelmsUniversität zu Bonn, Agrarwissenschaften, Diss., 1995

BRODERICK, G.A.; SATTER, L.D.; HARPER, A.E.:

Use of plasma amino acid concentration to identify limiting amino acids for milk production. J. Dairy Sci. 57 (1974), 1015-1023

BRUN-BELLUT, J.; BLANCHARD, G.; VIGNON, B.:

Effects of rumen-degradable protein concentration in diets on digestion, nitrogen utilization and milk yield by dairy goats. Small Ruminant Res. 3 (1990), 575-581

DE PETERS, E.J.; CANT, J.P.:

Nutritional factors influencing the nitrogen composition of bovine milk: a review. J. Dairy Sci. 75 (1992), 2043-2070

DLG-Futterwerttabellen Wiederkäuer, Hrsg. Universität Hohenheim, DLG-Verlag, Frankfurt, 7. Auflage, (1997)

HADJIPANAYIOTOU, M.:

Effect of feeding heat treated soyabean meal on the performance of lactating Damascus goats. Small Ruminant Res. 18 (1995), 105-111

HADJIPANAYIOTOU, M.; PHOTIOU, A.:

Effects of protein source and level on performance of lactating Damascus Goats in negative energy balance. Small Ruminant Res. 15 (1995), 257-263

HADJIPANAYIOTOU, M; KOUMAS, A.; HADJIGAVRIEL, G.; ANTONIOU, I; PHOTIOU, A.; THEODORIDOU, M:

Feeding dairy ewes and goats and growing lambs and kids mixtures of protein supplements. Small Ruminant Res. 21 (1996), 203-211

HADJIPANAYIOTOU, M.; MORAND-FEHR, P:

Intensive feeding of dairy goats In: Goat Nutrition. P. MORAND-FEHR (Ed.), Pudoc, Wageningen, pp 197-208 (1991)

HOFMANN, R.R.:

Evolutinary steps of ecophysiological adaptation and diversification of ruminants: a comparative view of their digestive system. Oecologica, 78 (1989), 443-457

Institut National de la Recherche Agronomique (INRA):

Alimentation des Bovins, Ovins et Caprins; INRA, Paris (1988)

Methodenbuch Bd. III:

Die chemische Untersuchung von Futtermitteln. Hsg. NAUMANN, C. und BASSLER, R., VDLUFA Verlag-Darmstadt; 1. Auflage 1976 und Ergänzungsauflagen (1997)

MISHRA, S.; RAI, S.N.:

Effects of different RDP and UDP ratios on voluntary intake, milk production and feed conversion efficiency in lactating goats. Small Ruminant Res. 20 (1996), 31-38

MORAND-FEHR, P. ; BOYAZOGLU, J.:

Present status and future outlook of the small ruminant sector. Small Ruminant Res. 34 (1999), 175-188

MORAND-FEHR, P.; SAUVANT, D.:

Nutrition and optimum performance of dairy goats. Livestock Prod. Sci. 5 (1978), 203-213

NRC:

Nutrient Requirements of goats: Angora, Dairy and meat goats in temperate and tropical countries.

National research Council, No. 15, (1981), 1-12

OLDHAM, J.D.: 
Amino acid metabolism in ruminants. Proc. Cornell Conf. for Feed Manufacturers, Cornell (USA). (1984), 137-151

PAILAN, G.H.; KAUR, H.:

Influence of dietary protein content and digestibility on milk yield and blood constituents in lactating goats. Small Ruminant Res. 20 (1996), 47-51

PAULICKS, B.:

Experimentelle Untersuchungen an laktierenden Kühen zu Veränderung von Milchmenge und Milchinhaltsstoffen bei Proteinfehlernährung und nachfolgend bedarfsgerechter Versorgung. Techn. Univ. München, Inst. f. Ernährungsphysiologie, Diss., 1985

RANAWANA, S.S.E.; KELLAWAY, R.C.:

Responses to postruminal infusions of graded levels of casein in lactating goats. Br. J. Nutr. 37 (1977a), 67-79

RANAWANA, S.S.E.; KELLAWAY, R.C.:

Responses to postruminal infusions of glucose and casein in lactating goats. Br. J. Nutr. 37 (1977b), 395-402

SAUVANT, D.; CHILIARD, Y.; MORAND-FEHR, P.:

Ethiological aspectsof nutritional and metabolic disorders of goats. In: Goat Nutrition. P. MORANDFEHR (Ed.), Pudoc, Wageningen, (1991), 124-142

SCHINGOETHE, D.J.:

Dietary influence on protein level in milk and milk yield in dairy cows. Animal Feed Sci. and Technol. 60 (1996), 181-190

SCHINGOETHE, D.J.; CASPER, D.P.; YANG, C.; ILLG, D.J.; SOMMERFELDT, J.L.; MUELLER, C.R.:

Lactational response to soybean meal, heated soybean meal and extruded soybeans with ruminally protected methionine. J. Dairy Sci. 71 (1988), 173-180

SUTTON, J.D. UND ALDERMAN, G.:

The energy and protein requirements of pregnant and lactating dairy goats The Agriculture and Food Research Council report Livestock Prod. Sci. 64 (2000), 3-8

TEWATIA, B.S.; KHATTA, V.K.; VIRK, A.S.; GUPTA, P.C.:

Effect of formaldehyde-treated faba beans (Vicia faba L.) on performance of lactating goats. Small Ruminant Res. 16 (1995), 107-111

TYRELL, H.F.; REID, J.T.:

Prediction of the energy value of cow’s milk. J. Dairy Sci. 48 (1965), 1215-1223

Eingegangen: 19.03.2002

Akzeptiert: 25.04.2002

Autor für Korrespondenz

Dr. CLAUDIA KIJORA

Humboldt Universität zu Berlin, Institut für Nutztierwissenschaften,

FG Tierzucht in den Tropen und Subtropen

Philippstr. 13, Haus 9

D-10115 Berlin

E-Mail: claudia.kijora@rz.hu-berlin.de oder k.peters@agrar.hu-berlin.de 